\title{
Harvesting Renewable Resources of Population with Size Structure and Diffusion
}

\author{
Qiang-Jun Xie, Ze-Rong He, and Chun-Guo Zhang \\ Institute of Operational Research and Cybernetics, Hangzhou Dianzi University, Hangzhou 310018, China \\ Correspondence should be addressed to Ze-Rong He; zrhe@hdu.edu.cn
}

Received 10 April 2014; Accepted 24 May 2014; Published 18 June 2014

Academic Editor: Abdul Latif

Copyright (C) 2014 Qiang-Jun Xie et al. This is an open access article distributed under the Creative Commons Attribution License, which permits unrestricted use, distribution, and reproduction in any medium, provided the original work is properly cited.

The aim of this work is to explore the optimal exploitation way for a biological resources model incorporating individual's size difference and spatial effects. The existence of a unique nonnegative solution to the state system is shown by means of Banach's fixed point theorem, and the continuous dependence of the population density with the harvesting effort is given. The optimal harvesting strategy is established via normal cone and adjoint system technique. Some conditions are found to assure that there is only one optimal policy.

\section{Introduction and Problem Setting}

Since the classical work by Skellam [1], dispersal or diffusion of biological individuals has been recognized as one of the most significant features, which affect the dynamics and evolution of populations. Researchers made lots of efforts to understand the role of dispersal in the distribution of populations and the structure of communities; see, for example, $[2,3]$. On the other hand, size-structured models are still an active field due to their ecological importance and mathematical challenge (see, e.g., $[4,5]$ ).

This paper is concerned with an optimal harvesting problem of a species model, which incorporates the dispersal and body size of organisms. There are a number of results in some particular situations, such as [6-8] for ageand space-structured models and [9-18] for size-structured models without consideration of diffusion. In [9], Botsford constructed a size-specific population model based on the continuity equation and suggested that the inclusion of individual growth rates could reveal optimal harvesting policies. Some linear optimal harvesting population models structured by size were introduced in $[10,11]$. The optimality conditions of Pontryagin's type were obtained in [12] for an optimal control problem for a size-structured system. Hritonenko et al. [13] analyzed nonlinear optimal control of integral-differential equations that described the optimal management of a forest; Gasca-Leyva et al. [14] analyzed the optimal harvesting time for husbanded biological assets consisting of individuals of different sizes. In [15], Davydov and Platov established size-structured population dynamics in the case where the growth rate, mortality, and exploitation intensity depend only on the size. The global stability of a nontrivial stationary state and some necessary optimality conditions were obtained. Kato in [16] sought the optimal harvesting rate in a profit maximization problem for a nonlinear size-structured model of two-species population. Other nonlinear size-specific models can be found in $[17,18]$. He and Liu [17] took fertility as the control variable and established the necessary optimality conditions of first order in the form of an Euler-Lagrange system. The existence of a unique optimal controller was established by means of Ekeland's variational principle. Similar techniques were introduced in [18], and the optimality conditions describing the optimal strategy were also obtained via tangent-normal cone technique.

There are, however, only few models that combine size structure with spatial diffusion. In Section 1.2 of [5], Webb introduced a kind of population models with size structure and spatial position. The theory of semigroup of operators was employed to study the existence and asymptotical behaviors; some numerical simulations were made as an aid to 
understand the theoretical results. Moreover, a tumor growth model was examined. Faugeras and Maury established an advection model of fish with length and plane position distribution in [19], for which the well-posedness was rigorously treated and approximation procedure presented. Furthermore, the model was applied to skipjack tuna population in the Indian Ocean. Hadeler (in [20]) emphasized the value of introducing diffusion to structured population models and discussed how to supply appropriate boundary conditions for the models.

The objective of this paper is to investigate an optimal harvesting problem for a size- and space-structured population model and to analyze the structure of the optimal strategies. We are motivated to optimize the economic profits functional of resources exploitation as follows:

$$
\begin{gathered}
\operatorname{maximize} J(u):=\int_{\underline{s}}^{\bar{s}} \int_{0}^{T} \int_{\Omega}\left[w(s, t, x) u(s, t, x) p^{u}(s, t, x)\right. \\
\left.\quad-\frac{1}{2} \rho u^{2}(s, t, x)\right] d x d t d s,
\end{gathered}
$$

subject to $u \in \mathcal{U}=\left\{v \in L^{2}(Q): 0 \leq \zeta_{1}(s, t, x) \leq v(s, t, x) \leq\right.$ $\zeta_{2}(s, t, x)$ a.e. in $\left.Q\right\}$, where $Q:=(\underline{s}, \bar{s}) \times(0, T) \times \Omega, T>0$ is the finite horizon of control, and $\Omega \subset R^{N}(N \leq 3)$ is a bounded open domain with a boundary $\partial \Omega$ smooth enough. Constants $\underline{s}$ and $\bar{s}$ stand for, respectively, the minimal and maximal size of individuals. $w(s, t, x)$ denotes the economic value of an individual of size $s \in[\underline{s}, \bar{s}]$ at time $t \in[0, T]$ at location $x \in \Omega$. $\rho>0$ is a costs factor for implementing the control policy $u$. $p^{u}(s, t, x)$ is the population density corresponding to $u$, which is governed by the following system:

$$
\begin{gathered}
\frac{\partial p}{\partial t}+\frac{\partial(g(s) p)}{\partial s}-k \Delta p=-\mu(s, t, x) p-u(s, t, x) p, \quad(s, t, x) \in Q, \\
g(\underline{s}) p(\underline{s}, t, x)=\int_{\underline{s}}^{\bar{s}} \beta(s, t, x) p(s, t, x) d s, \\
\left.p(s, 0, x)=p_{0}(s, x), \quad(s, x) \in(0, T) \times \Omega, \bar{s}\right) \times \Omega, \\
\frac{\partial p}{\partial n}(s, t, x)=0, \quad(s, t, x) \in \Sigma,
\end{gathered}
$$

where $\Sigma:=(\underline{s}, \bar{s}) \times(0, T) \times \partial \Omega, n$ is the outward unit normal, and the system (2) is endowed with the homogeneous Neumann boundary condition, which means no exchange of population across $\partial \Omega$. Control variable $u \in \mathcal{U}$ is the harvesting effort; functions $\zeta_{1}(s, t, x)$ and $\zeta_{2}(s, t, x)$ give the minimum and maximum harvesting efforts, respectively. The vital parameters $\mu(s, t, x)$ and $\beta(s, t, x)$ are the death and birth rates, $k>0$ is the diffusive coefficient, and $p_{0}(s, x)$ is the initial size and spatial distribution of our target population. $g(s)$ is the growth modulus of size; that is, $g(s)=d s / d t$.

Hereafter, let $\Gamma(s):=\int_{s}^{s}(1 / g(v)) d v$. We need the following definition.
Definition 1. The set of curves $S=\{(s, t) \in(\underline{s}, \bar{s}) \times(0, T)$ : $t-\Gamma(s)=h, h \in R\}$ is called the family of characteristic curves.

Throughout this paper, we make the following hypotheses:

$\left(\mathrm{H}_{1}\right) g \in C^{1}[\underline{s}, \bar{s}], g(\underline{s})>0$, and $0 \leq g(s) \leq \bar{g} \leq 2 g(\bar{s})$ for $\forall s \in[\underline{s}, \bar{s}]$

$\left(\mathrm{H}_{2}\right) \mu \in L_{\mathrm{loc}}^{\infty}([\underline{s}, \bar{s}) \times[0, T] \times \bar{\Omega}), \mu(s, t, x) \geq \mu_{0}(s, t) \geq 0$, a.e. in $Q$, where $\mu_{0} \in L_{\mathrm{loc}}^{\infty}([\underline{s}, \bar{s}) \times[0, T])$ and $\int_{\underline{s}}^{\bar{s}} \mu_{0}(s, t-$ $\Gamma(\bar{s})+s) d s=+\infty$, a.e. $t \in[0, T]$;

$\left(\mathrm{H}_{3}\right) \beta(s, t, x) \geq 0$ a.e. in $Q, \beta \in L^{\infty}(Q)$, and let $\bar{\beta}:=$ $\|\beta\|_{L^{\infty}(\mathrm{Q})}$;

$\left(\mathrm{H}_{4}\right) p_{0}(s, x) \geq 0$ a.e. in $(\underline{s}, \bar{s}) \times \Omega, p_{0} \in L^{\infty}((\underline{s}, \bar{s}) \times \Omega)$, and let $\bar{p}_{0}:=\left\|p_{0}\right\|_{L^{\infty}((\underline{s}, \bar{s}) \times \Omega)}$;

$\left(\mathrm{H}_{5}\right) w(s, t, x)>0$ a.e. in $Q$ from $(1), w \in L^{\infty}(Q)$, and let $W:=\|w\|_{L^{\infty}(\mathrm{Q})}$.

Denote by $D p$ the directional derivative operator of $p$; that is,

$$
D p(s, t, x):=\lim _{h \rightarrow 0} \frac{p\left(\Gamma^{-1}(\Gamma(s)+h), t+h, x\right)-p(s, t, x)}{h} .
$$

It is obvious that $D p=\partial p / \partial t+g(s)(\partial p / \partial s)$ for $p$ smooth enough.

Define

$$
\begin{aligned}
A C & \left(S ; L^{2}(\Omega)\right) \\
& :=\left\{k: S \longrightarrow L^{2}(\Omega) ; k\right. \text { is absolutely }
\end{aligned}
$$

continous on any compact subinterval\}.

Then, we introduce the definition of weak solutions as follows.

Definition 2. By solution of system (2), we mean a function $p(s, t, x) \in L^{2}(Q)$, which belongs to $C\left(\bar{S} ; L^{2}(\Omega)\right) \cap$ $A C\left(S ; L^{2}(\Omega)\right) \cap L^{2}\left(S ; H^{1}(\Omega)\right) \cap L_{\text {loc }}^{2}\left(S ; H^{2}(\Omega)\right)$ for almost any characteristic curve $S$ and satisfies

$$
\begin{gathered}
D p(s, t, x)-k \Delta p=-\left[\mu(s, t, x)+u(s, t, x)+g^{\prime}(s)\right] p, \\
\text { a.e. }(s, t, x) \in Q, \\
\lim _{h \rightarrow 0+} g\left(\Gamma^{-1}(h)\right) p\left(\Gamma^{-1}(h), t+h, \cdot\right) \\
=\int_{\underline{s}}^{\bar{s}} \beta(s, t, \cdot) p(s, t, \cdot) d s, \quad \text { in } L^{2}(\Omega), \text { a.e. } t \in(0, T), \\
\lim _{h \rightarrow 0+} p\left(\Gamma^{-1}(\Gamma(s)+h), h, \cdot\right)=p_{0}(s, \cdot), \\
\text { in } L^{2}(\Omega), \quad \text { a.e. } s \in(\underline{s}, \bar{s}), \\
\frac{\partial p}{\partial n}(s, t, x)=0, \quad \text { a.e. }(s, t, x) \in \Sigma .
\end{gathered}
$$


The remainder of this paper is organized as follows. The next section deals with the well-posedness of the state system (2) for given parameters. Section 3 derives the necessary optimality conditions and describes an optimal feedback law of control, while Section 4 establishes the existence and uniqueness of optimal strategies. The paper ends with a remarks section.

\section{Well-Posedness of the State System}

Lemma 3. If $\mu \in L^{\infty}(Q)$ and hypotheses $\left(H_{1}\right),\left(H_{3}\right)$, and $\left(H_{4}\right)$ hold, then the system (2) has a unique nonnegative solution.

Proof. Without loss of generality, we may assume that $u(s, t, x) \equiv 0$. Let $b(t, x) \in L^{2}((0, T) \times \Omega)$ be the density of population at the minimum size $\underline{s}$, and denote by $p_{b}$ the solution of the following system:

$$
\begin{gathered}
D p(s, t, x)-k \Delta p=-\left[\mu(s, t, x)+g^{\prime}(s)\right] p, \quad(s, t, x) \in Q, \\
g(\underline{s}) p(\underline{s}, t, x)=b(t, x), \quad(t, x) \in(0, T) \times \Omega, \\
p(s, 0, x)=p_{0}(s, x), \quad(s, x) \in(\underline{s}, \bar{s}) \times \Omega, \\
\frac{\partial p}{\partial n}(s, t, x)=0, \quad(s, t, x) \in \Sigma .
\end{gathered}
$$

In what follows, we prove the existence and uniqueness of $p_{b}$ following the spirits in [6].

Firstly, we replace $p$ in the right-hand side of the first equation in (6) with $q \in L^{2}(Q)$ arbitrarily fixed:

$$
\begin{gathered}
D p-k \Delta p=-\left(\mu+g^{\prime}\right) q, \quad(s, t, x) \in Q, \\
g(\underline{s}) p(\underline{s}, t, x)=b(t, x), \quad(t, x) \in(0, T) \times \Omega, \\
p(s, 0, x)=p_{0}(s, x), \quad(s, x) \in(\underline{s}, \bar{s}) \times \Omega, \\
\frac{\partial p}{\partial n}(s, t, x)=0, \quad(s, t, x) \in \Sigma .
\end{gathered}
$$

Then, we regard the above problem as a collection of linear parabolic systems on the characteristic curves $S$ (let $\left(s_{0}, t_{0}\right)$ be an initial point of $S$ ):

$$
\begin{aligned}
& \frac{\partial \widetilde{p}_{S}}{\partial h}-k \Delta \widetilde{p}_{S}=-\left(\widetilde{\mu}(h, x)+g^{\prime}\left(\Gamma^{-1}\left(\Gamma\left(s_{0}\right)+h\right)\right)\right) \tilde{q}_{S}(h, x), \\
& (h, x) \in(0, \alpha) \times \Omega, \\
& \frac{\partial \widetilde{p}_{S}}{\partial n}(h, x)=0, \quad(h, x) \in(0, \alpha) \times \partial \Omega, \\
& \tilde{p}_{S}(0, x)= \begin{cases}\frac{b\left(t_{0}, x\right)}{g(\underline{s})}, & \text { if } s=s_{0}, \quad x \in \Omega . \\
p_{0}\left(s_{0}, x\right), & \text { if } t=t_{0},\end{cases}
\end{aligned}
$$

Here, $\widetilde{p}_{S}(h, x):=p_{b, q}\left(\Gamma^{-1}\left(\Gamma\left(s_{0}\right)+h\right), t_{0}+h, x\right)$ and $p_{b, q}$ is the solution to (7), which is a linear heat equation. By standard theory of PDE (see, e.g., [21]), we assure that the system (7) has a unique solution $p_{b, q} \in L^{2}(Q)$. Clearly, $p_{b, q} \in$ $C\left(\bar{S} ; L^{2}(\Omega)\right) \cap A C\left(S ; L^{2}(\Omega)\right) \cap L^{2}\left(S ; H^{1}(\Omega)\right) \cap L_{\text {loc }}^{2}\left(S ; H^{2}(\Omega)\right)$ for almost any characteristic curve $S$.

Secondly, by Banach's fixed point theorem, we infer that there exists a unique bounded solution $p_{b}$ to the system (6). The proof is trivial and is omitted here.

We now define an operator $\mathscr{A}: L^{2}((0, T) \times \Omega) \rightarrow$ $L^{2}((0, T) \times \Omega)$ as

$$
(\mathscr{A} b)(t, x):=\int_{\underline{s}}^{\bar{s}} \beta(s, t, x) p_{b}(s, t, x) d s, \quad(t, x) \in(0, T) \times \Omega .
$$

Consider a norm in space $L^{2}((0, T) \times \Omega)$ given by

$$
\|v\|=\left(\int_{0}^{T} e^{-\lambda t}\|v(t, \cdot)\|_{L^{2}(\Omega)}^{2} d t\right)^{1 / 2}, \quad \forall v \in L^{2}((0, T) \times \Omega) .
$$

Clearly, it is equivalent to the usual norm. The constant $\lambda$ will be determined later.

Let $b_{1}, b_{2} \in L^{2}((0, T) \times \Omega)$, and $r(s, t, x)=p_{b_{1}}(s, t, x)-$ $p_{b_{2}}(s, t, x)$. It can be readily verified that $r$ is the solution of the following system:

$$
\begin{gathered}
\frac{\partial r}{\partial t}+\frac{\partial(g(s) r)}{\partial s}+\mu r-k \Delta r=0, \quad(s, t, x) \in Q, \\
g(\underline{s}) r(\underline{s}, t, x)=b_{1}(t, x)-b_{2}(t, x), \quad(t, x) \in(0, T) \times \Omega, \\
r(s, 0, x)=0, \quad(s, x) \in(\underline{s}, \bar{s}) \times \Omega, \\
\frac{\partial r}{\partial n}(s, t, x)=0, \quad(s, t, x) \in \Sigma .
\end{gathered}
$$

Multiplying the first equation in (11) by $r$ and integrating over $Q_{t}:=(\underline{s}, \bar{s}) \times(0, t) \times \Omega$, we get that

$$
\begin{aligned}
& \int_{\Omega} \int_{\underline{s}}^{\bar{s}} \int_{0}^{t} r \frac{\partial r}{\partial \tau} d \tau d s d x+\int_{\Omega} \int_{0}^{t} \int_{\underline{s}}^{\bar{s}} r \frac{\partial(g(s) r)}{\partial s} d s d \tau d x \\
& =-\int_{Q_{t}}\left(\mu r^{2}-k r \Delta r\right) d \sigma,
\end{aligned}
$$

where by $\int_{\mathrm{Q}_{t}}(\cdot) d \sigma$ we mean $\int_{\underline{s}}^{\bar{s}} \int_{0}^{t} \int_{\Omega}(\cdot) d x d \tau d s$; the style will be used hereafter.

By $\left(\mathrm{H}_{1}\right)$, we obtain that

$$
\begin{aligned}
& \int_{\Omega} \int_{0}^{t} \int_{\underline{s}}^{\bar{s}} r \frac{\partial(g(s) r)}{\partial s} d s d \tau d x \\
& \quad=\int_{\Omega} \int_{0}^{t}\left\{\left.\left[g(s) r^{2}(s, \tau, x)\right]\right|_{\underline{s}} ^{\bar{s}}-\int_{\underline{s}}^{\bar{s}} g(s) r \frac{\partial r}{\partial s} d s\right\} d \tau d x \\
& \quad \geq \int_{\Omega} \int_{0}^{t}\left\{\left.\left[g(s) r^{2}(s, \tau, x)\right]\right|_{\underline{s}} ^{\bar{s}}-\int_{\underline{s}}^{\bar{s}} \bar{g} r \frac{\partial r}{\partial s} d s\right\} d \tau d x
\end{aligned}
$$




$$
\begin{aligned}
& =\int_{\Omega} \int_{0}^{t}\left\{\left.\left[g(s) r^{2}(s, \tau, x)\right]\right|_{\underline{s}} ^{\bar{s}}-\left.\frac{1}{2} \bar{g} r^{2}(s, \tau, x)\right|_{\underline{s}} ^{\bar{s}}\right\} d \tau d x \\
& \geq \int_{\Omega} \int_{0}^{t}\left[\left(g(\bar{s})-\frac{1}{2} \bar{g}\right) r^{2}(\bar{s}, \tau, x)-g(\underline{s}) r^{2}(\underline{s}, \tau, x)\right] d \tau d x \\
& \geq-\int_{\Omega} \int_{0}^{t} g(\underline{s}) r^{2}(\underline{s}, \tau, x) d \tau d x .
\end{aligned}
$$

In addition, we are able to write down that

$$
\int_{\Omega} \int_{\underline{s}}^{\bar{s}} \int_{0}^{t} r \frac{\partial r}{\partial \tau} d \tau d s d x=\frac{1}{2}\|r(\cdot, t, \cdot)\|_{\left.L^{2}(\underline{s}, \bar{s}) \times \Omega\right)}^{2} .
$$

So we have

$$
\|r(\cdot, t, \cdot)\|_{L^{2}((\underline{s}, \bar{s}) \times \Omega)}^{2} \leq 2 \int_{0}^{t} \int_{\Omega} g(\underline{s}) r^{2}(\underline{s}, t, x) d x d \tau .
$$

Then, we can derive the following inequalities:

$$
\begin{aligned}
& \left\|\mathscr{A} b_{1}-\mathscr{A} b_{2}\right\|^{2} \\
& =\int_{0}^{T} e^{-\lambda t}\left\|\int_{\underline{s}}^{\bar{s}} \beta(s, t, \cdot)\left(p_{b_{1}}-p_{b_{2}}\right)(s, t, \cdot) d s\right\|_{L^{2}(\Omega)}^{2} d t \\
& \leq \bar{\beta} \int_{0}^{T} e^{-\lambda t}\left\|\int_{\underline{s}}^{\bar{s}} r(s, t, \cdot) d s\right\|_{L^{2}(\Omega)}^{2} d t \\
& \leq(\bar{s}-\underline{s}) \bar{\beta} \int_{0}^{T} e^{-\lambda t}\|r(\cdot, t, \cdot)\|_{L^{2}((\underline{s}, \bar{s}) \times \Omega)}^{2} d t \\
& \leq 2(\bar{s}-\underline{s}) \bar{\beta} \int_{0}^{T} e^{-\lambda t} \int_{\Omega} g(\underline{s}) r^{2}(\underline{s}, t, x) d x d t \\
& \leq \frac{2(\bar{s}-\underline{s}) \bar{\beta}}{g(\underline{s})} \int_{0}^{T} \int_{0}^{t} e^{-\lambda t}\left\|b_{1}(\tau, \cdot)-b_{2}(\tau, \cdot)\right\|_{L^{2}(\Omega)}^{2} d \tau d t \\
& =\frac{2(\bar{s}-\underline{s}) \bar{\beta}}{g(\underline{s})} \int_{0}^{T} \int_{\tau}^{T} e^{-\lambda t}\left\|b_{1}(\tau, \cdot)-b_{2}(\tau, \cdot)\right\|_{L^{2}(\Omega)}^{2} d t d \tau \\
& \leq \frac{2(\bar{s}-\underline{s}) \bar{\beta}}{g(\underline{s})} \frac{1}{\lambda} \int_{0}^{T} e^{-\lambda t}\left\|b_{1}(t, \cdot)-b_{2}(t, \cdot)\right\|_{L^{2}(\Omega)}^{2} d t \\
& =\frac{2(\bar{s}-\underline{s}) \bar{\beta}}{g(\underline{s})} \frac{1}{\lambda}\left\|b_{1}-b_{2}\right\|^{2} \text {. }
\end{aligned}
$$

It is now obvious that, for any $\lambda>2(\bar{s}-\underline{s}) \bar{\beta} / g(\underline{s}), \mathscr{A}$ is a contraction on $\left(L^{2}((0, T) \times \Omega),\|\cdot\|\right)$. Banach's fixed point theorem allows us to conclude that there exists a unique $b^{*} \in$ $L^{2}((0, T) \times \Omega)$ such that $b^{*}=\mathscr{A} b^{*}$. Let

$$
\begin{gathered}
L_{+}^{2}((0, T) \times \Omega):=\left\{v(t, x) \in L^{2}((0, T) \times \Omega) ; v(t, x) \geq 0\right. \\
\text { a.e. in }(0, T) \times \Omega\} .
\end{gathered}
$$

Since $\mathscr{A}\left(L_{+}^{2}((0, T) \times \Omega)\right) \subset L_{+}^{2}((0, T) \times \Omega)$ and $L_{+}^{2}((0, T) \times$ $\Omega)$ is closed in $L^{2}((0, T) \times \Omega)$, then $b^{*}(t, x) \in L_{+}^{2}((0, T) \times$ $\Omega)$. Consequently, $p_{b^{*}}(s, t, x)$ is the desired solution of system (2).

Using the approximating procedure of Banach's fixed point, we obtain the following monotonicity result.

Lemma 4. Under the hypotheses of Lemma 3, let $p_{i}(i=1,2)$ be the solutions of the system (2) corresponding to $\left(\mu_{i}, \beta_{i}, p_{0 i}\right)$. If $\mu_{1} \geq \mu_{2}, \beta_{1} \leq \beta_{2}, p_{01} \leq p_{02}$, then

$$
0 \leq p_{1}(s, t, x) \leq p_{2}(s, t, x) \quad \text { a.e. in } Q \text {. }
$$

Then, we prove the main result of this section.

Theorem 5. Under the hypotheses $\left(H_{1}\right)-\left(H_{4}\right)$, system (2) has a unique solution $p(s, t, x)$ in $Q$. Furthermore, the solution is nonnegative and bounded:

$$
0 \leq p(s, t, x) \leq M_{1} \quad \text { a.e. in } Q
$$

where $M_{1}=\|\bar{p}\|_{L^{\infty}(\mathrm{Q})}$ and $\bar{p}$ is the solution of system (2) with $\mu=0, \beta=\bar{\beta}$, and $p_{0}=\bar{p}_{0}$.

Proof. For any $N \in \mathbf{N}^{*}$ (the set of all positive integers), we define

$$
\mu^{N}(s, t, x):=\min \{\mu(s, t, x), N\}, \quad(s, t, x) \in Q .
$$

It is apparent that $\mu^{N}$ satisfies the assumption of $\mu$ in Lemma 3 , and the sequence $\left\{\mu^{N}\right\}$ is increasing. Denote by $p_{N}$ the unique nonnegative solution of system (2) corresponding to $\mu^{N}$. For $N_{1} \leq N_{2} \leq N_{3} \leq \cdots$, we have $\mu^{N_{1}} \leq \mu^{N_{2}} \leq \mu^{N_{3}} \leq$ $\cdots$, and so $p_{N_{1}} \geq p_{N_{2}} \geq p_{N_{3}} \geq \cdots \geq 0$ by Lemma 4 . Beppo Levi's theorem implies that

$$
p_{N}(s, t, x) \longrightarrow p(s, t, x) \text { a.e. in } Q \text {, as } N \longrightarrow+\infty \text {. }
$$

So $p(s, t, x) \geq 0$ a.e. in $Q$. It is not difficult to prove that $p$ is the unique solution of system (2) (see, e.g., Theorem 4.1.3 in [6]), but we omit the details.

Then, we examine the boundedness of solutions. Since $\bar{p}$ is the unique nonnegative solution of (2) corresponding to $\mu=0, \beta=\bar{\beta}$, and $p_{0}=\bar{p}_{0}$, where $\bar{\beta}$ and $\bar{p}_{0}$ are constants given in $\left(\mathrm{H}_{3}\right)$ and $\left(\mathrm{H}_{4}\right)$, Lemma 4 implies that

$$
0 \leq p(s, t, x) \leq \bar{p} \quad \text { a.e. in } Q \text {. }
$$

Letting $M_{1}=\|\bar{p}\|_{L^{\infty}(\mathrm{Q})}$ leads to

$$
0 \leq p(s, t, x) \leq M_{1} \quad \text { a.e. in } Q
$$

which completes the proof.

\section{Optimal Feedback Policy}

In order to establish our main theorem, we need the following auxiliary results. 
Lemma 6. Let $p^{u_{1}}, p^{u_{2}}$ be the solutions of system (2) corresponding to the controls $u_{1}, u_{2} \in \mathcal{U}$, respectively. Then, one has

$$
\left\|p^{u_{1}}-p^{u_{2}}\right\|_{L^{2}(\mathrm{Q})}^{2} \leq T C_{1}\left\|u_{1}-u_{2}\right\|_{L^{2}(\mathrm{Q})}^{2},
$$

where $C_{1}$ is a constant independent of $u_{1}, u_{2}$.

Proof. Let $y=p^{u_{1}}-p^{u_{2}}$. Then, $y$ is the solution of

$$
\begin{gathered}
\frac{\partial y}{\partial t}+\frac{\partial(g(s) y)}{\partial s}=k \Delta y-\mu y-u_{1} y-\left(u_{1}-u_{2}\right) p^{u_{2}}, \\
(s, t, x) \in Q, \\
g(\underline{s}) y(\underline{s}, t, x)=\int_{\underline{s}}^{\bar{s}} \beta(s, t, x) y(s, t, x) d s, \\
(t, x) \in(0, T) \times \Omega, \\
y(s, 0, x)=0, \quad(s, x) \in(\underline{s}, \bar{s}) \times \Omega, \\
\frac{\partial y}{\partial n}(s, t, x)=0, \quad(s, t, x) \in \Sigma .
\end{gathered}
$$

Multiplying the first equation of (25) by $y$, integrating on $Q_{t}$, we obtain

$$
\begin{aligned}
& \int_{\Omega} \int_{\underline{s}}^{\bar{s}} \int_{0}^{t} y \frac{\partial y}{\partial \tau} d \tau d s d x+\int_{\Omega} \int_{0}^{t} \int_{\underline{s}}^{\bar{s}} y \frac{\partial(g(s) y)}{\partial s} d s d \tau d x \\
& =\int_{Q_{t}} k y \Delta y d \sigma-\int_{Q_{t}}\left(\mu+u_{1}\right) y^{2} d \sigma \\
& \quad+\int_{Q_{t}}\left(u_{2}-u_{1}\right) p^{u_{2}} y d \sigma .
\end{aligned}
$$

Proceeding in a similar way to the proof of Lemma 3, we arrive at

$$
\begin{aligned}
&\|y(\cdot, t, \cdot)\|_{L^{2}((\underline{s}, \bar{s}) \times \Omega)}^{2} \\
& \leq 2 \int_{0}^{t} \int_{\Omega} g(\underline{s}) y^{2}(\underline{s}, \tau, x) d x d \tau+2 \int_{Q_{t}}\left(u_{2}-u_{1}\right) p^{u_{2}} y d \sigma \\
& \leq \frac{2}{g(\underline{s})} \int_{0}^{t} \int_{\Omega}\left(\int_{\underline{s}}^{\bar{s}} \beta(s, \tau, x) y(s, \tau, x) d s\right) d x d \tau \\
&+\int_{0}^{t}\|y(\cdot, \tau, \cdot)\|_{L^{2}((\underline{s}, \bar{s}) \times \Omega)}^{2} d \tau \\
&+M_{1}^{2} \int_{Q_{t}}\left|u_{1}(s, \tau, x)-u_{2}(s, \tau, x)\right| d \sigma \\
& \leq\left(1+C^{*}\right) \int_{0}^{t}\|y(\cdot, \tau, \cdot)\|_{L^{2}((\underline{s}, \bar{s}) \times \Omega)}^{2} d \tau \\
&+M_{1}^{2} \int_{Q_{t}}\left|u_{1}(s, \tau, x)-u_{2}(s, \tau, x)\right|^{2} d \sigma,
\end{aligned}
$$

where $M_{1}$ is as in Theorem 5 and $C^{*}:=2 \bar{\beta}^{2}(\bar{s}-\underline{s}) / g(\underline{s})$. Bellman's lemma implies that

$$
\|y(\cdot, t, \cdot)\|_{L^{2}((\underline{s}, \bar{s}) \times \Omega)}^{2} \leq M_{1}^{2} e^{\left(1+C^{*}\right) T}\left\|u_{1}-u_{2}\right\|_{L^{2}(Q)}^{2}
$$

holds for any $t \in(0, T)$. Consequently,

$$
\|y\|_{L^{2}(\mathrm{Q})}^{2} \leq T M_{1}^{2} e^{\left(1+C^{*}\right) T}\left\|u_{1}-u_{2}\right\|_{L^{2}(\mathrm{Q})}^{2} .
$$

Then, letting $C_{1}:=M_{1}^{2} e^{\left(1+C^{*}\right) T}$ gives the conclusion of Lemma 6.

We now define the following dual problem associated with the system (2):

$$
\begin{gathered}
\frac{\partial q}{\partial t}+g(s) \frac{\partial q}{\partial s}+k \Delta q=(\mu+u) q+w u-q(\underline{s}) \beta \\
(s, t, x) \in Q \\
\frac{\partial q}{\partial n}(s, t, x)=0, \quad(s, t, x) \in \Sigma, \\
q(s, T, x)=0, \quad(s, x) \in(\underline{s}, \bar{s}) \times \Omega, \quad(t, x) \in(0, T) \times \Omega . \\
q(\bar{s}, t, x)=0,
\end{gathered}
$$

Denote by $q^{u}(s, t, x)$ the solution to (30) corresponding to $u \in$ $\mathcal{U}$. Introducing the transformation $\widehat{q}(\bar{s}-s, T-t, x)=q(s, t, x)$, we then treat the system (30) in the same manner as that in Theorem 5 and Lemma 6 and obtain the properties of $q$ as follows.

Lemma 7. The dual system (30) has a unique solution $q^{u} \in$ $L^{\infty}(Q)$ and

$$
\left|q^{u}(s, t, x)\right| \leq M_{2} \quad \text { a.e. in } Q
$$

where $M_{2}$ is a positive constant.

Furthermore, let $q^{u_{1}}, q^{u_{2}}$ be the solutions of the system (30) corresponding to $u_{1}, u_{2} \in \mathcal{U}$, respectively. Then, there exists a positive constant $C_{2}$, which is independent of $u_{1}, u_{2}$, such that

$$
\left\|q^{u_{1}}-q^{u_{2}}\right\|_{L^{2}(\mathrm{Q})}^{2} \leq T C_{2}\left\|u_{1}-u_{2}\right\|_{L^{2}(\mathrm{Q})}^{2} .
$$
result.

By a standard reasoning, we may derive the following

Lemma 8. Suppose that $u^{*} \in \mathcal{U}$ is a solution for the optimal control problem (1), and $p^{u^{*}}$ is the corresponding solution of system (2). Then, for any $v \in L^{\infty}(Q)$, such that $u^{*}+\varepsilon v \in \mathcal{U}$ for $\varepsilon>0$ small enough, the following holds:

$$
\frac{1}{\varepsilon}\left(p^{u^{*}+\varepsilon v}-p^{u^{*}}\right) \longrightarrow z, \quad \text { a.e. in } Q \text { as } \varepsilon \longrightarrow 0^{+}
$$


where $z \in L^{\infty}(Q)$ satisfies

$$
\begin{gathered}
\frac{\partial z}{\partial t}+\frac{\partial(g(s) z)}{\partial s}-k \Delta z=-\left(\mu+u^{*}\right) z-v p^{u^{*}}, \\
(s, t, x) \in Q, \\
g(\underline{s}) z(\underline{s}, t, x)=\int_{\underline{s}}^{\bar{s}} \beta(s, t, x) z(s, t, x) d s, \\
z(s, 0, x) \in(0, T) \times \Omega, \quad(s, x) \in(\underline{s}, \bar{s}) \times \Omega, \\
\frac{\partial z}{\partial n}(s, t, x)=0, \quad(s, t, x) \in \Sigma .
\end{gathered}
$$

We now establish the structure of optimal controllers in a feedback form.

Theorem 9. Let $u^{*} \in \mathcal{U}$ be an optimal policy for problem (1)(2) and $p^{u^{*}}$ and $q$ the corresponding solutions of systems (2) and (30). Then,

$$
u^{*}(s, t, x)=\mathscr{F}\left\{\frac{[w+q] p^{u^{*}}}{\rho}\right\}(s, t, x),
$$

in which the mapping $\mathscr{F}$ is defined as

$$
\begin{aligned}
& (\mathscr{F} h)(s, t, x) \\
& \quad= \begin{cases}\zeta_{1}(s, t, x), & h(s, t, x)<\zeta_{1}(s, t, x), \\
h(s, t, x), & \zeta_{1}(s, t, x) \leq h(s, t, x) \leq \zeta_{2}(s, t, x), \\
\zeta_{2}(s, t, x), & h(s, t, x)>\zeta_{2}(s, t, x) .\end{cases}
\end{aligned}
$$

Proof. Let $\mathscr{T}_{\mathcal{U}}\left(u^{*}\right)$ be the tangent cone to $\mathcal{U}$ at $u^{*}$ (see [22]). For any $v \in \mathscr{T}_{u}\left(u^{*}\right)$, we know that $u^{*}+\varepsilon v \in \mathcal{U}$ for $\varepsilon>0$ small enough. Since $u^{*}$ is optimal, it follows that

$$
\begin{aligned}
& \int_{Q}\left(w u^{*} p^{u^{*}}-\frac{1}{2} \rho u^{* 2}\right) d x d t d s \\
& \quad \geq \int_{Q}\left(w\left(u^{*}+\varepsilon v\right) p^{u^{*}+\varepsilon v}-\frac{1}{2} \rho\left(u^{*}+\varepsilon v\right)^{2}\right) d x d t d s,
\end{aligned}
$$

which implies that

$$
\begin{aligned}
\int_{Q}\left(w u^{*}\right. & \frac{p^{u^{*}+\varepsilon v}-p^{u^{*}}}{\varepsilon}+w v p^{u^{*}+\varepsilon v} \\
& \left.-\frac{1}{2} \rho v\left(2 u^{*}+\varepsilon v\right)\right) d x d t d s \leq 0 .
\end{aligned}
$$

Passing to limit $\varepsilon \rightarrow 0^{+}$in (38) and using Lemma 8 , we have

$$
\int_{Q} w u^{*} z d x d t d s+\int_{Q} v\left(w p^{u^{*}}-\rho u^{*}\right) d x d t d s \leq 0
$$

where $z$ is the solution of the system (34).
In order to derive the optimality conditions, we will use the structure of the corresponding normal cone. Firstly, we verify the following integral relation:

$$
\int_{Q} w u^{*} z d x d t d s=\int_{Q} v p^{u^{*}} q d x d t d s
$$

Actually, multiplying the first equation of (30) by $z(s, t, x)$, integrating on $Q$, and using (34), we obtain that

$$
\begin{aligned}
& \int_{Q} w u^{*} z d x d t d s \\
& =\int_{\underline{s}}^{\bar{s}} \int_{\Omega} \int_{0}^{T} \frac{\partial q}{\partial t} z d t d x d s+\int_{0}^{T} \int_{\Omega} \int_{\underline{s}}^{\bar{s}} g(s) \frac{\partial q}{\partial s} z d s d x d t \\
& +\int_{Q} k z \Delta q d x d t d s \\
& =-\int_{\underline{s}}^{\bar{s}} \int_{\Omega} \int_{0}^{T} q \frac{\partial z}{\partial t} d t d x d s \\
& -\int_{0}^{T} \int_{\Omega} g(\underline{s}) z(\underline{s}, t, x) q(\underline{s}, t, x) d x d t \\
& -\int_{0}^{T} \int_{\Omega} \int_{\underline{s}}^{\bar{s}} q \frac{\partial(g(s) z)}{\partial s} d s d x d t+\int_{Q} k q \Delta z d x d t d s \\
& =-\int_{Q} q\left(\frac{\partial z}{\partial t}+\frac{\partial(g(s) z)}{\partial s}-k \Delta z\right) d x d t d s \\
& -\int_{Q} q(\underline{s}, t, x) \beta(s, t, x) z(s, t, x) d x d t d s, \\
& \int_{Q} v p^{u^{*}} q d x d t d s \\
& =\int_{Q}\left(\mu+u^{*}\right) q z d x d t d s+\int_{Q} w u^{*} z d x d t d s \\
& -\int_{Q} q(\underline{s}, t, x) \beta(s, t, x) z(s, t, x) d x d t d s .
\end{aligned}
$$

So we have

$$
\begin{aligned}
\int_{Q} & \left(\left(\mu+u^{*}\right) q z+w u^{*} z\right) d x d t d s \\
= & -\int_{Q} q\left(\frac{\partial z}{\partial t}+\frac{\partial(g(s) z)}{\partial s}-k \Delta z\right) d x d t d s
\end{aligned}
$$

Similarly, it follows from the system (34) that

$$
\begin{gathered}
\int_{Q} q\left(\frac{\partial z}{\partial t}+\frac{\partial(g(s) z)}{\partial s}-k \Delta z\right) d x d t d s \\
=-\int_{Q}\left(\left(\mu+u^{*}\right) z q+v p^{u^{*}} q\right) d x d t d s
\end{gathered}
$$


The relations (42) and (43) imply that (40) is true. Then, from (38) and (40), it follows that

$$
\begin{array}{r}
\int_{Q}\left\{\left[(w+q) p^{u^{*}}-\rho u^{*}\right] v\right\}(s, t, x) d x d t d s \leq 0, \\
\forall v \in \mathscr{T}_{\mathcal{U}}\left(u^{*}\right) .
\end{array}
$$

Therefore, according to the properties of normal cone (see [22]), the expression in the square brackets of (44) satisfies $(w+q) p^{u^{*}}-\rho u^{*} \in \mathcal{N}_{\mathcal{u}}\left(u^{*}\right)$, the normal cone to $\mathcal{U}$ at $u^{*}$. Consequently, the conclusion follows.

\section{Existence of a Unique Optimal Policy}

In this section, we prove the existence and uniqueness of optimal policies. The main result reads as follows.

Theorem 10. Let the hypotheses $\left(H_{1}\right)-\left(H_{5}\right)$ be satisfied. If

$$
T<\frac{\rho^{2}}{\left(W\left(1+M_{2}\right) \sqrt{C_{1}}+W M_{1} \sqrt{C_{2}}\right)^{2}}
$$

where $W$ is as in $\left(H_{5}\right)$ and constants $M_{i}, C_{i}(i=1,2)$ are given in Theorem 5 and Lemmas 6 and 7, then the optimal harvesting problem (1)-(2) has a unique solution.

Proof. Firstly, we define a function of $\varepsilon \in(0,1)$ as

$$
H(\varepsilon):=J\left(\varepsilon u_{1}+(1-\varepsilon) u_{2}\right)
$$

where $J(\cdot)$ is of the form (1) and $u_{1}$ and $u_{2} \in \mathcal{U}$ are arbitrarily fixed. We will show that $H^{\prime}(\varepsilon)$ is strictly decreasing, and then $J(u)$ is strictly concave in $\mathcal{U}$.

Denote by $p^{\varepsilon}, p^{\varepsilon+\delta}(0<\delta \ll 1)$ the solutions of $(2)$ corresponding to $\varepsilon u_{1}+(1-\varepsilon) u_{2}$ and $(\varepsilon+\delta) u_{1}+(1-(\varepsilon+\delta)) u_{2}$, respectively. It follows from (1) that

$$
\begin{aligned}
& H^{\prime}(\varepsilon) \\
& =\lim _{\delta \rightarrow 0} \frac{1}{\delta}[H(\varepsilon+\delta)-H(\varepsilon)] \\
& =\lim _{\delta \rightarrow 0} \frac{1}{\delta}\left\{\int _ { Q } \left\{w\left[(\varepsilon+\delta) u_{1}+(1-(\varepsilon+\delta)) u_{2}\right] p^{\varepsilon+\delta}\right.\right. \\
& \left.-\frac{1}{2} \rho\left[(\varepsilon+\delta) u_{1}+(1-(\varepsilon+\delta)) u_{2}\right]^{2}\right\} d \sigma \\
& -\int_{Q}\left\{w\left[\varepsilon u_{1}+(1-\varepsilon) u_{2}\right] p^{\varepsilon}\right. \\
& \left.\left.-\frac{1}{2} \rho\left[\varepsilon u_{1}+(1-\varepsilon) u_{2}\right]^{2}\right\} d \sigma\right\}
\end{aligned}
$$

$$
\begin{aligned}
& =\lim _{\delta \rightarrow 0} \frac{1}{\delta} \int_{Q}\left\{w\left[\varepsilon u_{1}+(1-\varepsilon) u_{2}\right]\left(p^{\varepsilon+\delta}-p^{\varepsilon}\right)\right. \\
& \left.+w \delta\left(u_{1}-u_{2}\right) p^{\varepsilon+\delta}\right\} d \sigma \\
& -\rho \int_{Q}\left[\varepsilon u_{1}+(1-\varepsilon) u_{2}\right]\left(u_{1}-u_{2}\right) d \sigma \\
& =\int_{Q}\left[w\left(u_{2}+\varepsilon\left(u_{1}-u_{2}\right)\right) z^{\varepsilon}+w\left(u_{1}-u_{2}\right) p^{\varepsilon}\right] d \sigma \\
& \quad-\int_{Q}\left[\rho\left(u_{2}+\varepsilon\left(u_{1}-u_{2}\right)\right)\left(u_{1}-u_{2}\right)\right] d \sigma,
\end{aligned}
$$

where $z^{\varepsilon}$ is the solution of (34) corresponding to $u_{2}+\varepsilon\left(u_{1}-\right.$ $\left.u_{2}\right)$. By the same argument as that in Theorem 9, we have

$$
\int_{Q} w\left(u_{2}+\varepsilon\left(u_{1}-u_{2}\right)\right) z^{\varepsilon} d \sigma=\int_{Q}\left(u_{1}-u_{2}\right) p^{\varepsilon} q^{\varepsilon} d \sigma
$$

where $q^{\varepsilon}$ is the solution of (30) corresponding to $u_{2}+\varepsilon\left(u_{1}-\right.$ $u_{2}$ ). Therefore,

$$
\begin{aligned}
& H^{\prime}(\varepsilon) \\
& \quad=\int_{Q}\left(u_{1}-u_{2}\right)\left[w p^{\varepsilon}\left(1+q^{\varepsilon}\right)-\rho\left(u_{2}+\varepsilon\left(u_{1}-u_{2}\right)\right)\right] d \sigma .
\end{aligned}
$$

Next, choosing $\varepsilon_{1}, \varepsilon_{2} \in(0,1)$ and $\varepsilon_{1} \neq \varepsilon_{2}$, we get that

$$
\begin{aligned}
& {\left[H^{\prime}\left(\varepsilon_{1}\right)-H^{\prime}\left(\varepsilon_{2}\right)\right]\left(\varepsilon_{1}-\varepsilon_{2}\right)} \\
& =\left(\varepsilon_{1}-\varepsilon_{2}\right) \int_{Q}\left(u_{1}-u_{2}\right)\left[w p^{\varepsilon_{1}}\left(1+q^{\varepsilon_{1}}\right)-w p^{\varepsilon_{2}}\left(1+q^{\varepsilon_{2}}\right)\right] d \sigma \\
& \quad-\rho\left(\varepsilon_{1}-\varepsilon_{2}\right)^{2} \int_{Q}\left(u_{1}-u_{2}\right)^{2} d \sigma \\
& =\left(\varepsilon_{1}-\varepsilon_{2}\right) \int_{Q} w\left(u_{1}-u_{2}\right)\left[\left(p^{\varepsilon_{1}}-p^{\varepsilon_{2}}\right)\left(1+q^{\varepsilon_{1}}\right)\right. \\
& \quad-\rho\left(\varepsilon_{1}-\varepsilon_{2}\right)^{2} \int_{Q}\left(u_{1}-u_{2}\right)^{2} d \sigma \\
& \left.\left.\quad:=I_{1}-\rho\left(\varepsilon_{1}-\varepsilon_{2}\right)^{2} \int_{Q}^{\varepsilon_{1}}-q^{\varepsilon_{2}}\right)\right] d \sigma
\end{aligned}
$$

Combining Theorem 5 and Lemmas 6 and 7 with CauchySchwarz inequality, it follows from $\left(\mathrm{H}_{5}\right)$ that

$$
\begin{aligned}
I_{1} \leq\left|\varepsilon_{1}-\varepsilon_{2}\right| W\{ & \left(1+M_{2}\right) \int_{Q}\left|u_{1}-u_{2}\right|\left|p^{\varepsilon_{1}}-p^{\varepsilon_{2}}\right| d \sigma \\
& \left.+M_{1} \int_{Q}\left|u_{1}-u_{2}\right|\left|q^{\varepsilon_{1}}-q^{\varepsilon_{2}}\right| d \sigma\right\}
\end{aligned}
$$




$$
\begin{aligned}
\leq & W\left(1+M_{2}\right)\left|\varepsilon_{1}-\varepsilon_{2}\right| \\
& \times\left(\int_{Q}\left|u_{1}-u_{2}\right|^{2} d \sigma \cdot \int_{Q}\left|p^{\varepsilon_{1}}-p^{\varepsilon_{2}}\right|^{2} d \sigma\right)^{1 / 2} \\
& +W M_{1}\left|\varepsilon_{1}-\varepsilon_{2}\right|\left(\int_{Q}\left|u_{1}-u_{2}\right|^{2} d \sigma \cdot \int_{Q}\left|q^{\varepsilon_{1}}-q^{\varepsilon_{2}}\right|^{2} d \sigma\right)^{1 / 2} \\
\leq & {\left[W\left(1+M_{2}\right) \sqrt{T C_{1}}+W M_{1} \sqrt{T C_{2}}\right] } \\
& \times\left(\varepsilon_{1}-\varepsilon_{2}\right)^{2} \int_{Q}\left|u_{1}-u_{2}\right|^{2} d \sigma \\
= & \sqrt{T}\left(W\left(1+M_{2}\right) \sqrt{C_{1}}+W M_{1} \sqrt{C_{2}}\right) \\
& \times\left(\varepsilon_{1}-\varepsilon_{2}\right)^{2} \int_{Q}\left|u_{1}-u_{2}\right|^{2} d \sigma .
\end{aligned}
$$

Consequently,

$$
\begin{aligned}
& {\left[H^{\prime}\left(\varepsilon_{1}\right)-H^{\prime}\left(\varepsilon_{2}\right)\right]\left(\varepsilon_{1}-\varepsilon_{2}\right)} \\
& \leq\left(\sqrt{T}\left(W\left(1+M_{2}\right) \sqrt{C_{1}}+W M_{1} \sqrt{C_{2}}\right)-\rho\right)\left(\varepsilon_{1}-\varepsilon_{2}\right)^{2} \\
& \quad \times \int_{Q}\left(u_{1}-u_{2}\right)^{2} d x d t d s .
\end{aligned}
$$

If $T<\rho^{2} /\left(W\left(1+M_{2}\right) \sqrt{C_{1}}+W M_{1} \sqrt{C_{2}}\right)^{2}$, then we have

$$
\left[H^{\prime}\left(\varepsilon_{1}\right)-H^{\prime}\left(\varepsilon_{2}\right)\right]\left(\varepsilon_{1}-\varepsilon_{2}\right)<0 .
$$

Hence, $H^{\prime}(\varepsilon)$ is strictly decreasing, which implies the strict concavity of $J(u)$ in $\mathcal{U}$.

Define the functional $\phi: L^{2}(Q) \rightarrow[-\infty,+\infty)$ as follows:

$$
\phi(u)= \begin{cases}J(u), & \text { if } u \in \mathcal{U}, \\ -\infty, & \text { if } u \notin \mathcal{U} .\end{cases}
$$

It is clear that $\phi$ is concave in $L^{2}(Q)$. By Lemma 6, we claim that $\phi$ is upper semicontinuous. Since $\mathcal{U}$ is convex, closed, and bounded and $J(u)$ is strictly concave, $J(u)$ attains its unique maximum in $\mathcal{U}$, which is the solution to problem (1)-(2). The proof is completed.

\section{Concluding Remarks}

As a main research result in the present paper, Theorem 9 describes the law of optimal harvesting, which is given by a feedback manner in terms of the corresponding state and costate variables. The results in this paper may serve as a base to many realistic applications. In those situations, one must estimate the parameters in the harvesting problem, such as price function $w(s, t, x)$, growth function $g(s)$, and vital rates $\mu, \beta$, and then apply some proper numerical procedure or algorithm to obtain an approximating solution with errors small enough. We should be satisfied with such treatment since the coupled state-costate system cannot be expected to get analytical solutions.

\section{Disclosure}

All authors have seen the paper and agreed to submit it to this journal.

\section{Conflict of Interests}

The authors declare that there is no conflict of interests regarding the publication of this paper.

\section{Acknowledgments}

The authors thank the reviewers for their helpful comments and suggestions, which improved the paper very much. This research was supported by the National Natural Science Foundation of China (nos. 11271104, 41101508, and 61174074) and the Scientific Project of Educational Commission in Zhejiang Province of China (no. Y201327022).

\section{References}

[1] J. G. Skellam, "Random dispersal in theoretical populations," Biometrika, vol. 38, no. 1-2, pp. 196-218, 1951.

[2] A. Okubo and S. A. Levin, Diffusion and Ecological Problems: Modern Perspectives, Springer, New York, NY, USA, 2001.

[3] R. Nathan, H. Kokko, A. Lpez-Sepulcre et al., "Migration and Dispersal: Perspectives," Science, Special Section, vol. 313, no. 5788, pp. 786-795, 2006.

[4] B. Ebenman and L. Persson, Size-Structured Populations: Ecology and Evolution, Springer, Berlin, Germany, 1988.

[5] G. F. Webb, "Population models structured by age, size, and spatial position," in Structured Population Models in Biology and Epidemiology, P. Magal and S. Ruan, Eds., Lecture Notes in Math, pp. 1-49, Springer, Berlin, Germany, 2008.

[6] S. Aniţa, Analysis and Control of Age-Dependent Population Dynamics, Kluwer, Dordrecht, The Netherlands, 2000.

[7] C. Zhao, M. Wang, and P. Zhao, "Optimal harvesting problems for age-dependent interacting species with diffusion," Applied Mathematics and Computation, vol. 163, no. 1, pp. 117-129, 2005.

[8] Z. Luo, "Optimal harvesting problem for an age-dependent $n$ dimensional food chain diffusion model," Applied Mathematics and Computation, vol. 186, no. 2, pp. 1742-1752, 2007.

[9] L. W. Botsford, "Optimal fishery policy for size-specific, density-dependent population models," Journal of Mathematical Biology, vol. 12, no. 3, pp. 265-293, 1981.

[10] K. P. Hadeler and J. Müller, "Optimal harvesting and optimal vaccination," Mathematical Biosciences, vol. 206, no. 2, pp. 249272, 2007.

[11] N. Kato, "Maximum principle for optimal harvesting in linear size-structured population," Mathematical Population Studies, vol. 15, no. 2, pp. 123-136, 2008.

[12] O. C. Tarniceriu and V. M. Veliov, "Optimal control of a class of size-structured systems," in Large-Scale Scientific Computing, I. Lirkov, S. Margenov, and J. Wasniewski, Eds., vol. 4818 of Lecture Notes in Computer Science, pp. 366-373, Springer, Berlin, Germany, 2008.

[13] N. Hritonenko, Y. Yatsenko, R.-U. Goetz, and A. Xabadia, "Maximum principle for a size-structured model of forest and carbon sequestration management," Applied Mathematics Letters, vol. 21, no. 10, pp. 1090-1094, 2008. 
[14] E. Gasca-Leyva, J. M. Hernandez, and V. M. Veliov, "Optimal harvesting time in a sizeheterogeneous population," Ecological Modelling, vol. 210, no. 1-2, pp. 161-168, 2008.

[15] A. A. Davydov and A. S. Platov, "Optimization of stationary solution of a model of size-structured population exploitation," Journal of Mathematical Sciences, vol. 176, no. 6, pp. 860-869, 2011.

[16] N. Kato, "Optimal harvesting in a two-species model of sizestructured population," in Optimal Control of Age-Structured Populations in Economy, Demography, and the Environment, Boucekkine, N. Hritoenko, and Y. Yatsenko, Eds., pp. 229-252, Routledge Explorations in Environmental Economics, London, UK, 2011.

[17] Z.-R. He and Y. Liu, "An optimal birth control problem for a dynamical population model with size-structure," Nonlinear Analysis: Real World Applications, vol. 13, no. 3, pp. 1369-1378, 2012.

[18] Y. Liu, X.-L. Cheng, and Z.-R. He, "On the optimal harvesting of size-structured population dynamics," Applied Mathematics B: A Journal of Chinese Universities, vol. 28, no. 2, pp. 173-186, 2013.

[19] B. Faugeras and O. Maury, "An advection-diffusion-reaction size-structured fish population dynamics model combined with a statistical parameter estimation procedure: application to the Indian Ocean skipjack tuna fishery," Mathematical Biosciences and Engineering, vol. 2, no. 4, pp. 719-741, 2005.

[20] K. P. Hadeler, "Structured populations with diffusion in state space," Mathematical Biosciences and Engineering, vol. 7, no. 1, pp. 37-49, 2010.

[21] V. Barbu, Partial Differential Equations and Boundary Value Problems, Kluwer, Dordrecht, The Netherlands, 1998.

[22] J.-P. Aubin and I. Ekeland, Applied Nonlinear Analysis, John Wiley \& Sons, New York, NY, USA, 1984. 


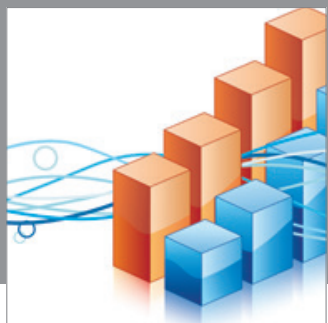

Advances in

Operations Research

mansans

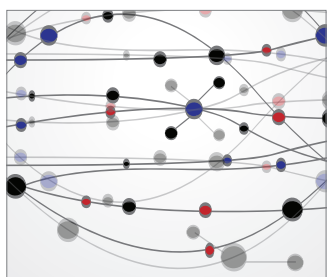

The Scientific World Journal
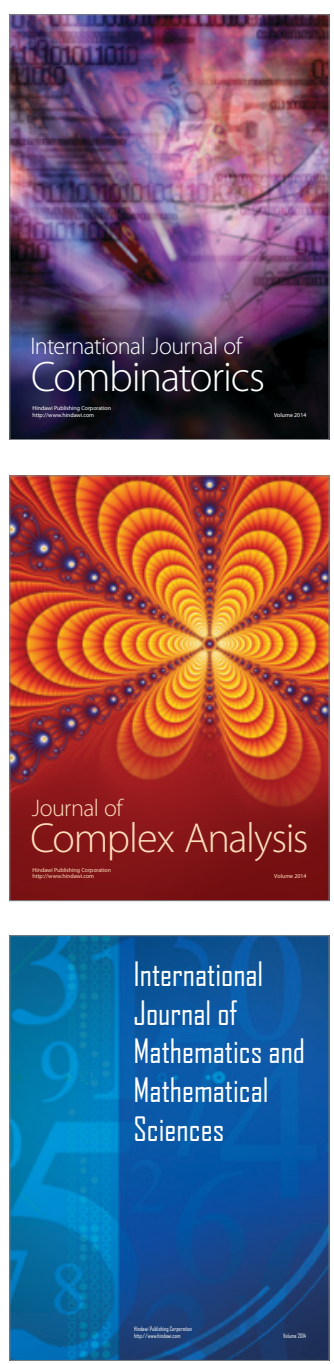
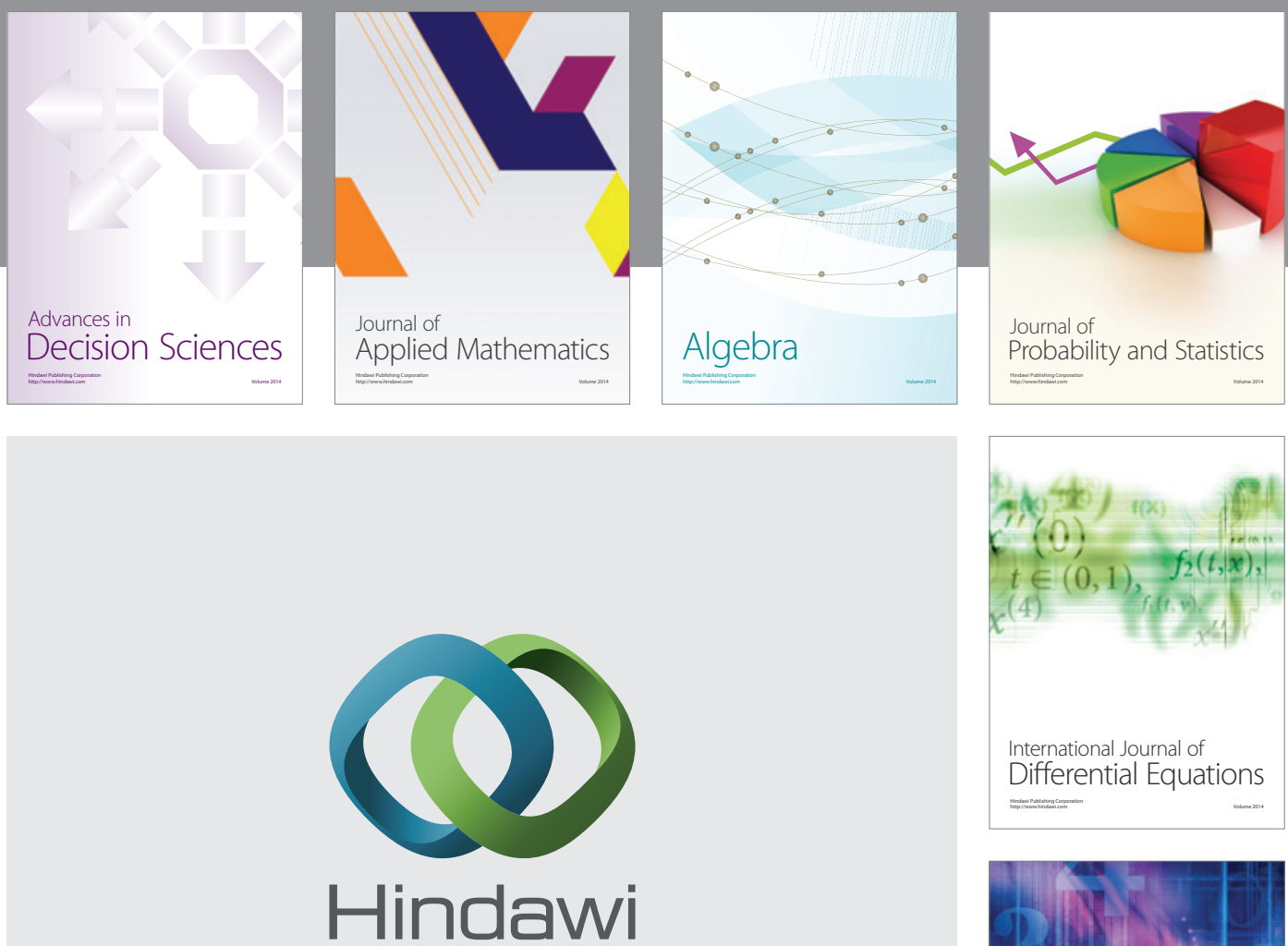

Submit your manuscripts at http://www.hindawi.com
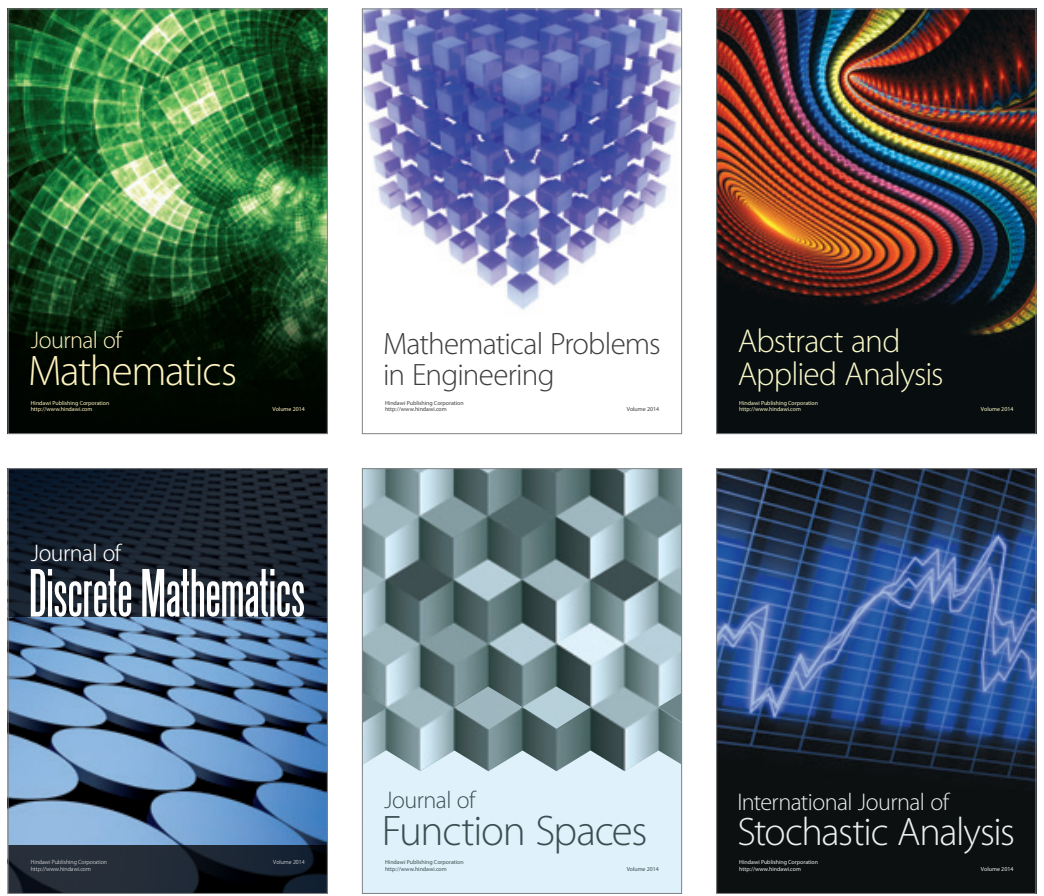

Journal of

Function Spaces

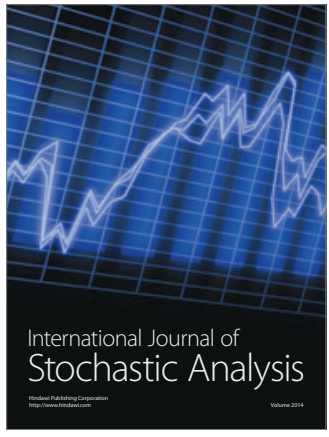

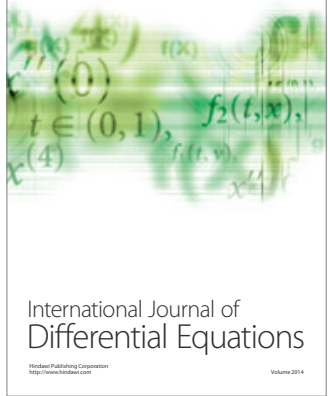
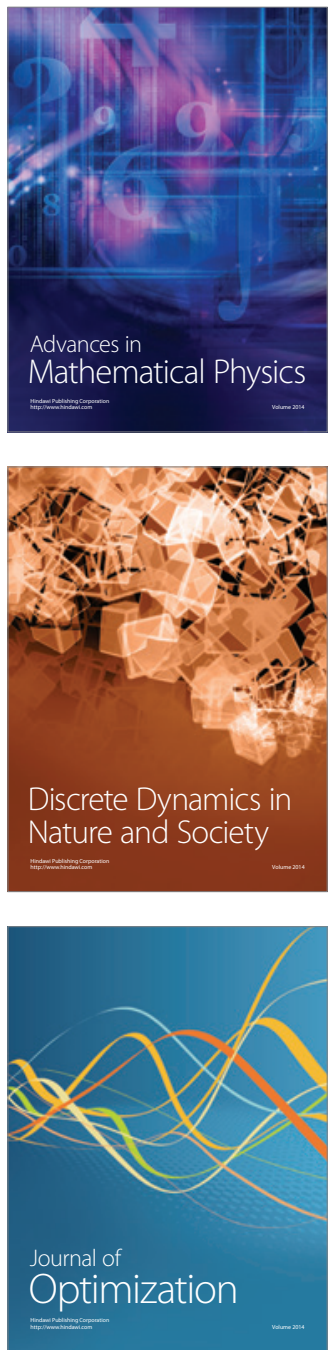